\title{
Vigencia de la operación de Hartmann en el tratamiento de la diverticulosis colónica complicada: estudio realizado en pacientes tratados durante el periodo 2009-2018, en Hospital de Clínicas de Montevideo
}

\author{
Validity of the Hartmann operation in the treatment of complicated colonic \\ diverticulosis: study carried out in patients treated during the period 2009-2018, at \\ Hospital de Clínicas, Montevideo
}

Carlos Barberousse ${ }^{1}$

ORCID https://orcid.org/0000-0001-9982-9369

Luciano Ramírez ${ }^{2}$

ORCID https://orcid.org/0000-0003-2493-0352

Santiago Dardanelli ${ }^{3}$

ORCID https://orcid.org/0000-0002-0885-3724

Lucía Fernández ${ }^{4}$

ORCID https://orcid.org/0000-0002-3051-7073

DOI 10.31837/cir.urug/5.2.3

Recibido: 7 de julio de 2020

Aceptado: 9 de marzo de 2021

\section{Resumen}

Introducción y objetivos: La enfermedad diverticular complicada constituye un problema de salud que afecta a pacientes añosos y muchas veces requiere tratamiento quirúrgico. Las opciones son múltiples, siendo la operación de Hartmann una alternativa antigua y aún vigente. El objetivo del presente estudio fue evaluar la vigencia de dicha intervención en nuestro medio a través del estudio analítico de una población en un Hospital universitario de Montevideo.

Materiales y métodos: Se analizaron las historias clínicas de los pacientes mayores de 20 años operados en el período 2009-2018. Se consideraron las siguientes variables: procedimientos quirúrgicos, complicaciones peri operatorias, re intervenciones, tasa y tiempo de reconstrucción del tránsito digestivo y mortalidad.

1.2.3.4 Clínica Quirúrgica A, Prof. Dr. Luis Cazabán, Hospital de Clínicas. Montevideo. Uruguay. Departamento de Emergencia del Hospital de Clínicas, Prof. Dr. Fernando Machado. Montevideo. Uruguay carbarbe@ hotmail.com 
Resultados: El número total de pacientes operados en el período analizado fue de 27, con una media de edad de 59,4 años. En 15 de ellos se realizó un procedimiento de Hartmann, En los restantes pacientes los procedimientos realizados fueron abocamiento de ambos cabos, anastomosis primaria con y sin estoma proximal y lavado y drenaje video-asistido sin resección. Presentaron complicaciones peri operatorias 11 pacientes $(40 \%)$. La mortalidad peri operatoria fue de cuatro pacientes $(14.8 \%)$, correspondiendo todos a la operación de Hartmann. La tasa de reconstrucción fue seis de 11 pacientes sobrevivientes.

Discusión: La operación de Hartmann mantiene vigencia y es el procedimiento más utilizado, siendo su morbimortalidad considerable y la tasa de reconstrucción de aproximadamente la mitad de los pacientes. La anastomosis primaria debe ser reservada para casos seleccionados. El desarrollo de la cirugía laparoscopia también ha permitido procedimientos conservadores, como el lavado y drenaje, evitando la resección en agudo, siendo esta opción una tendencia creciente.

Conclusiones: Se concluyó que la serie, si bien corta, reproduce lo revisado en la bibliografía.

Palabras claves: diverticulosis colónica, operación de Hartmann, clasificación de Hinchey, colostomía, reconstrucción.

\begin{abstract}
Introduction and objectives: Complicated diverticular disease is a health problem that affects elderly patients and often requires surgical treatment. Options are multiple, being Hartmann procedure an old and still valid alternative. The objective of the present study was to evaluate the validity of this procedure in our environment through an analytical study of a population in a University Hospital of Montevideo.

Materials and methods: Analyze the medical records of patients older than 20 years operated on in the period 2009-2018. Consider the following variables: surgical procedures, peri-operative complications, re-treatments, rate and time of reconstruction of the digestive transit and mortality.

Results: 27 patients were operated in the analyzed period, with a mean age of 59.4 years. In 15 of them, a Hartmann procedure was performed. In the remaining patients, procedures performed were approach both ends, primary anastomosis with or without a proximal stoma, and assisted video washing and drainage without resection. Eleven patients (40\%) presented peri-operative complications. Peri-operative mortality was four patients (14.8\%), all corresponding to Hartmann's operation. The recovery rate was six of 11 surviving patients.

Discussion: The Hartmann operation maintains the validity and the most used procedure, being its considerable morbidity and mortality and the recovery rate of approximately half of the patients. The primary anastomosis should be reserved for selected cases. The development of laparoscopic surgery has also allowed conservative procedures, such as washing and drainage, avoiding acute resection, this option being a growing trend.

Conclusions: It was concluded that the series, although short, reproduce what was reviewed in the bibliography.
\end{abstract}

Key words: colonic diverticulosis, Hartmann's operation, Hinchey's classification, colostomy, reconstruction. 
La enfermedad diverticular o diverticulosis, descrita por primera vez por Littre en 1700, es de alta prevalencia a nivel mundial siendo una carga importante para los sistemas de salud. Se define por la presencia de divertículos en el colon, predominantemente pseudodiverticulos adquiridos, conformados por las capas más internas del colon, mucosa y submucosa, que ante el aumento de la presión intraluminal atraviesan zonas débiles de la capa muscular, siendo revestidas por las serosa visceral.

De causa multifactorial, vinculada fundamentalmente a factores dietéticos, es infrecuente antes de los 40 años, aumentando su incidencia progresiva a partir de los 60 años ${ }^{1}$. Esto implica a pacientes añosos, en general con comorbilidades, lo que aumenta los riesgos ante la eventualidad de un tratamiento quirúrgico. Afortunadamente cursa en forma asintomática en más del $80 \%$ de los casos, y dentro de las formas sintomáticas, sólo un porcentaje menor presenta complicaciones graves ${ }^{2}$.

La forma más frecuente de presentación clínica se conoce como diverticulitis aguda, con dolor y defensa en el cuadrante inferior izquierdo del abdomen, vinculada al colon sigmoides, sitio de localización de más del $90 \%$ de los casos de diverticulosis. Esta entidad en general es controlada mediante tratamiento médico, basado en una dieta y la administración de antiinflamatorios y antibióticos, existiendo actualmente estudios que incluso cuestionan la utilidad de esto últimos ${ }^{3,4}$.

De las complicaciones, las más frecuentes son las infecciosas, que derivan de la solución de continuidad de algún divertículo.

Existen distintas formas de clasificar los grados de severidad de las complicaciones infecciosas,

\begin{tabular}{|c|c|c|c|}
\hline \multicolumn{2}{|r|}{ Finchey } & \multicolumn{2}{|r|}{ Finchey modificada } \\
\hline \multirow[t]{2}{*}{ I } & Absceso o flemón pericólico & 0 & Diverticulitis clínica leve \\
\hline & & Ia & $\begin{array}{c}\text { Pared colónica } \\
\text { engrosada inflamación }\end{array}$ \\
\hline \multirow[t]{2}{*}{ II } & $\begin{array}{l}\text { Absceso pélvico, intraabdominal o } \\
\text { retroperitoneal }\end{array}$ & & pericólica confinada \\
\hline & & $\mathrm{Ib}$ & $\begin{array}{l}\text { Absceso pericólico pequeño confinado } \\
\qquad(<5 \mathrm{~cm})\end{array}$ \\
\hline \multirow[t]{2}{*}{ III } & Peritonitis purulenta generalizada & II & $\begin{array}{l}\text { Absceso pelvico, intraabdominal } \\
\text { distante o retroperitoneal }\end{array}$ \\
\hline & & III & Penitonitis purulenta generalizada \\
\hline \multirow[t]{2}{*}{ IV } & Peritonitis fecaloidea & & \\
\hline & & IV & Peritonitis fecaloidea \\
\hline
\end{tabular}

Fig. 1 siendo la más frecuente y conocida la clasificación de Hinchey (fig. 1), con grados que van desde el absceso pericolónico a la peritonitis difusa fecaloidea.

La sospecha de una complicación infecciosa en general indica la realización de algún estudio imagenológico, de preferencia una Tomografía Axial Computada (TAC), para su confirmación y clasificación ${ }^{5}$. Otras complicaciones, de menor frecuencia, son el sangrado, en general auto limitado, la estenosis con suboclusión u oclusión, y la fistulización a órganos vecinos, siendo el

más frecuente la vejiga.

En cuanto al tratamiento, la presencia de una complicación en general requiere la internación del paciente. Las distintas opciones estarán guiadas por la TAC, dependiendo del grado de severidad ${ }^{6}$. Los grados I y II de Hinchey pueden manejarse en forma no operatoria, mediante la administración de antibióticos intravenosos y eventualmente, el drenaje percutáneo guiado por Ecografía o TAC, 
especialmente para abscesos mayores a los $4 \mathrm{~cm}$ de diámetro, que sean pasibles del mismo en función de la existencia de una ventana adecuada. En caso de no ser posible esto, o de presentar una evolución insatisfactoria, deberá optarse por un tratamiento operatorio. En caso de grados III y IV el tratamiento operatorio es mandatorio ${ }^{7}$.

De las opciones quirúrgicas, desde el surgimiento del procedimiento de Hartmann en 1921, la resección del segmento colónico afectado ha sido casi siempre la regla. Ello implica un tratamiento en dos tiempos: un primer tiempo de resección colónica, con abocamiento del cabo proximal como colostomía y cierre y abandono del cabo distal, y un segundo tiempo, no antes de tres o cuatro meses, para la restitución del tránsito, con anastomosis de los cabos. Otras opciones, como la cirugía en tres tiempos fueron quedando abandonadas ${ }^{8}$. En cuanto a la cirugía en un tiempo, es decir, la resección con anastomosis colónica primaria, se considera de elevado riesgo por la condición y el tipo de paciente en la que la misma se realiza. Solamente en casos muy seleccionados se opta por esta opción en la urgencia, dada su muy elevada morbimortalidad ${ }^{9}$. En casos de cirugías programadas, como pueden ser casos de fístulas o estenosis incompletas, la anastomosis primaria es el procedimiento de elección ${ }^{10}$.

Si bien el procedimiento de Hartmann sigue siendo el estándar en la mayoría de las series, como contrapartida se cuestiona que sólo se logra la restitución definitiva del tránsito en un número de pacientes que apenas supera la mitad ${ }^{11}$. Ello supone además de la afectación de la calidad de vida que representa la colostomía, ya sea transitoria o definitiva, la morbimortalidad asociada de ambos procedimientos.

El surgimiento y desarrollo de la cirugía laparoscópica ha planteado la opción del lavado peritoneal y drenaje, especialmente para pacientes con grado de Hinchey II y III, actuando como terapia puente para resolver la situación de urgencia, con eventual posterior cirugía resectiva con anastomosis primaria por igual abordaje, evitando los aspectos negativos del procedimiento de Hartmann. En el caso de Hinchey III, la principal condición para poder realizar este tratamiento no resectivo es la no identificación de una clara perforación colónica, en cuyo caso se recomienda siempre la resección del segmento afectado. Los resultados publicados son a veces contradictorios, y en general corresponden a centros de referencia, en pacientes seleccionados ${ }^{12}$.

El objetivo del presente trabajo es conocer cuál es la realidad del tratamiento quirúrgico de esta patología en nuestro hospital, durante un lapso de tiempo reciente de 10 años. Realizaremos un análisis descriptivo del tipo de pacientes, los distintos procedimientos efectuados y sus resultados precoces y tardíos. Compararemos dichos resultados con las publicaciones actuales al respecto estableciendo la vigencia o no del tradicional procedimiento de Hartmann como la opción predominante. 


\section{Pacientes y métodos}

El tipo de muestreo fue no probabilístico, de conveniencia, respetando los criterios de inclusión y exclusión.

Para evitar sesgos de selección se establecieron los siguientes criterios de inclusión: todos los pacientes mayores de 20 años, intervenidos quirúrgicamente con diagnóstico de enfermedad diverticular complicada en el Hospital de Clínicas de Montevideo, en el período 2009-2018. Dentro de las complicaciones se incluyeron las infecciosas, principalmente peritonitis y algunos abscesos pericolónicos, la estenosis con oclusión o suboclusión, las fístulas y el sangrado incoercible

Se excluyeron aquellos pacientes con diagnóstico de enfermedad diverticular complicada que no recibieron tratamiento, o que recibieron únicamente tratamiento médico, así como también aquellos pacientes menores de 20 años, dado el diferente patrón patogénico en este rango etario. También pacientes en los cuales no se pudo tener acceso a sus historias clínicas.

La recolección de datos se realizó mediante el análisis de historias clínicas, contando para ello con la asistencia del Departamento de metodología estadística del Hospital, lo cual nos permitió obtener el marco muestral que se utilizó como columna vertebral del trabajo. Se respetaron los principios éticos de la investigación en seres humanos mediante la obtención del consentimiento informado telefónico de los pacientes o de sus familiares en los casos que no estuvieron vivos, previo a la revisión de historias clínicas. Mediante encuestas telefónicas personales se completó la recolección de datos y actualización de los mismos. También se revisaron los registros de personas fallecidas. Se contó también con el asesoramiento y aval de la Comisión de Bioética del Hospital.

Como variables cuantitativas se utilizaron: la mortalidad operatoria (discreta), el número de complicaciones (discreta), número de re-intervenciones (discreta), tiempo de internación (continua) y tasa de pacientes en los que se reconstruyó el tránsito (discreta).

Como variables cualitativas se utilizaron: el tipo de complicaciones postoperatorias (nominal) y ocurrencia de re-intervenciones (nominal).

Las complicaciones se analizaron en función de la clasificación Clavien-Dindo (C-D) con su última modificación (Anexo 1) 13,14

Para valorar las variables cuantitativas utilizamos media, varianza y desvío estándar. Para valorar las variables cualitativas se utilizaron medidas de frecuencias y gráficos. En algunos casos con un nivel de significancia de un 5\% se utilizaron test estadísticos como chi cuadrado o test $\mathrm{t}$ student comparando variables. Para realizar el procesamiento de datos cualitativos se utilizó el software profesional SPSS.

\section{Resultados}

Durante el período 2009-2018 (diez años) en el Hospital de Clínicas fueron intervenidos quirúrgicamente un total de 27 pacientes por complicaciones de una enfermedad diverticular. La

DOI: 10.31837/cir.urug/5.2.3 Cir. Urug. 2021 Vol. 5 No. 2 jul-dic 2021 1-17 
media de edad de los pacientes fue de 59.4 años, correspondiendo 15 (55.6\%) al sexo femenino y 12 al sexo masculino $(44.4 \%)$.

Del total de pacientes, $24(88,9 \%)$ fueron operados de urgencia y tres $(11,1 \%)$ lo fueron de coordinación.

Dentro de los 24 operados de urgencia, 22 pacientes presentaban complicaciones infecciosas $(81,48 \%)$. Tomando en cuenta la clasificación de Hinchey, de estos pacientes, en un caso $(4,5 \%)$ fue Hinchey I, tres casos $(13,6 \%)$ Hinchey II, 14 (63,6\%) Hinchey III, y cuatro $(18,3 \%)$ Hinchey IV (Tabla 1).

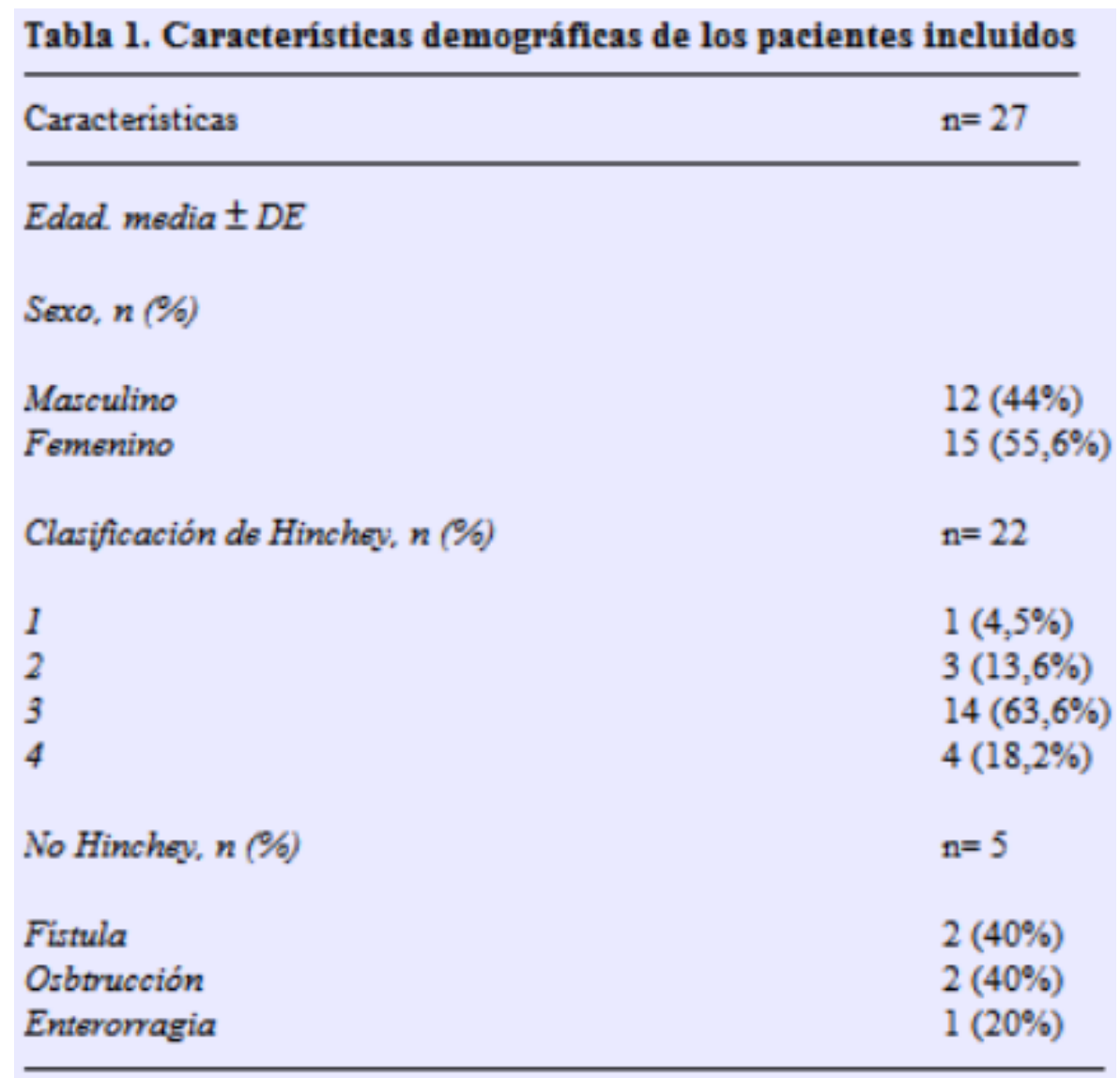

\section{$D E$ : desvio estandar}

De estos 22 pacientes, en 17 de los casos se realizó una resección del sigmoides. En 13 de ellos se completó el procedimiento con una operación de Hartmann, abocando el cabo proximal como una colostomía terminal, con cierre y abandono del cabo distal en el abdomen. En los cuatro pacientes restantes, en tres de ellos se realizó una anastomosis primaria, con una colostomía proximal de detransitación en un caso, y en el restante se realizó una colostomía a dos cabos “en caño de escopeta". En los cinco pacientes restantes operados por complicaciones infecciosas se realizó un abordaje laparoscópico, con lavado peritoneal y drenaje del foco, sin realizar una resección sigmoidea. Destacamos que estos cinco pacientes correspondieron a los grados de Hinchey I y II (total cuatro pacientes) y el restante fue un paciente con un grado III en el que no se identificó el sitio de la perforación sigmoidea.

Los dos pacientes operados de urgencia por complicaciones no infecciosas, en un caso se trató de una estenosis sigmoidea con oclusión, en el cual también se realizó una resección sigmoidea con un 
procedimiento de Hartmann, y el caso restante se trató de una enterorragia con repercusión hemodinámica, con igual procedimiento quirúrgico. (Gráfico 1)

\section{Gráfico 1}

Título

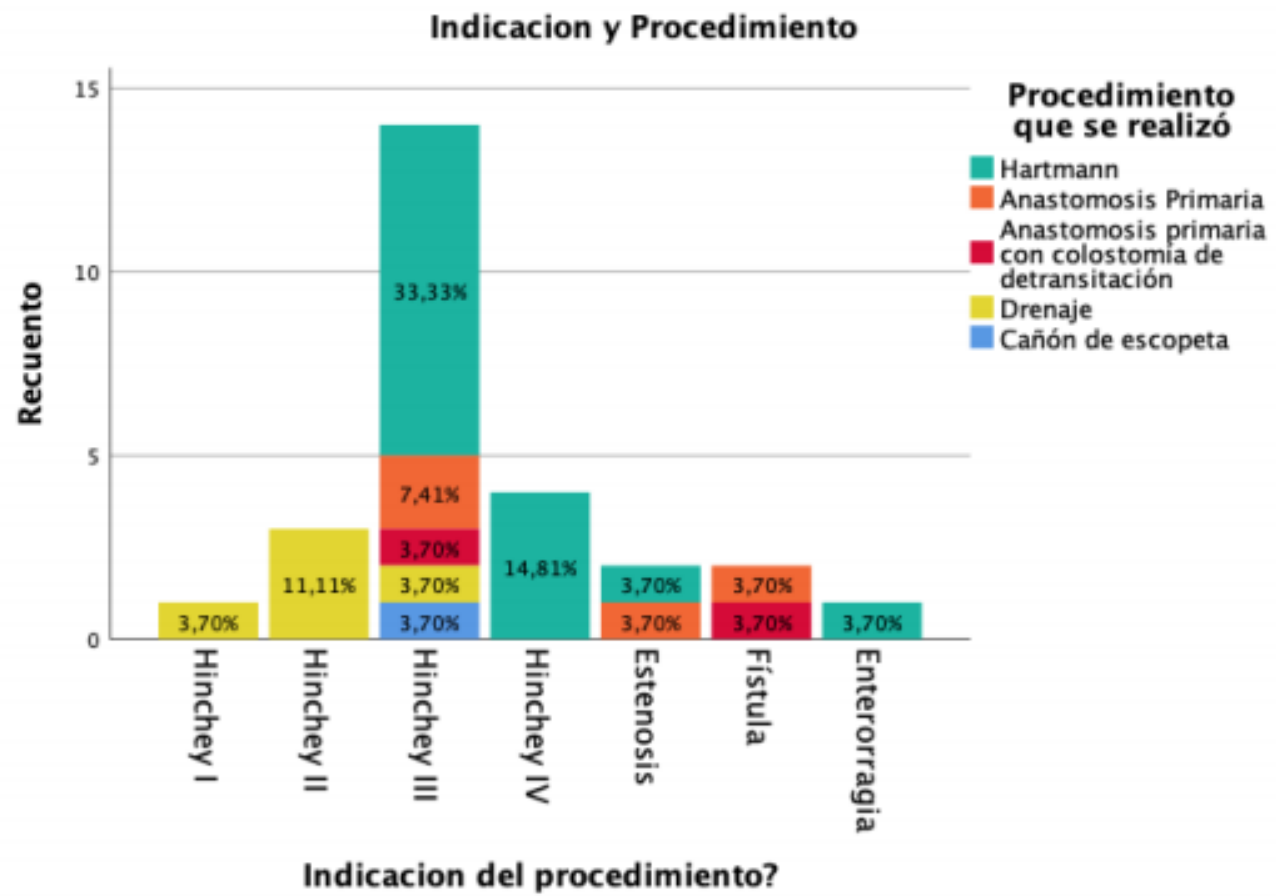

\section{Gráf. 1}

Del total de los 24 pacientes operados de urgencia, en 19 se realizó una resección sigmoidea, 15 de ellos con un procedimiento de Hartmann (62.5\%), tres con anastomosis primaria (12.5\%), siendo en un caso protegida dicha anastomosis con una colostomía proximal, y el restante paciente con una colostomía a dos cabos (4.1\%). Cinco de 24 pacientes intervenidos de urgencia fueron por abordaje laparoscópico $(20.8 \%)$, con lavado peritoneal y drenaje.

De los tres pacientes operados en forma programada, dos casos correspondieron a fístulas colovesicales, en los cuales se realizó una puesta a plano de la fístula, con resección sigmoidea y anastomosis primaria, con cierre del orificio vesical e interposición de colgajo de epiplón. En uno de estos dos casos se realizó una colostomía proximal de detransitación. El restante paciente operado de coordinación se trató de una estenosis con suboclusión, en el cual se realizó una resección sigmoidea con anastomosis primaria. (Tabla 2)

\section{Tabla 2.}

$\underline{\text { Resección sin anastomosis }}$ $\mathbf{n}=16$

Hartmann $\mathrm{n}=15$

Cañón de escopeta $\mathrm{n}=1$

Resección con anastomosis $\quad n=6$

Anastomosis primaria $\quad \mathrm{n}=4$

Anastomosis con colostomia de detransitación $\quad \mathrm{n}=2$ 
En suma, del total de pacientes intervenidos, en un 55,6\% $(\mathrm{n}=15)$ de los casos se realizó procedimiento de Hartmann, con una media de edad fue 64,60 $\pm 12,26$. (Gráfico 2)

\section{Gráfico 2}

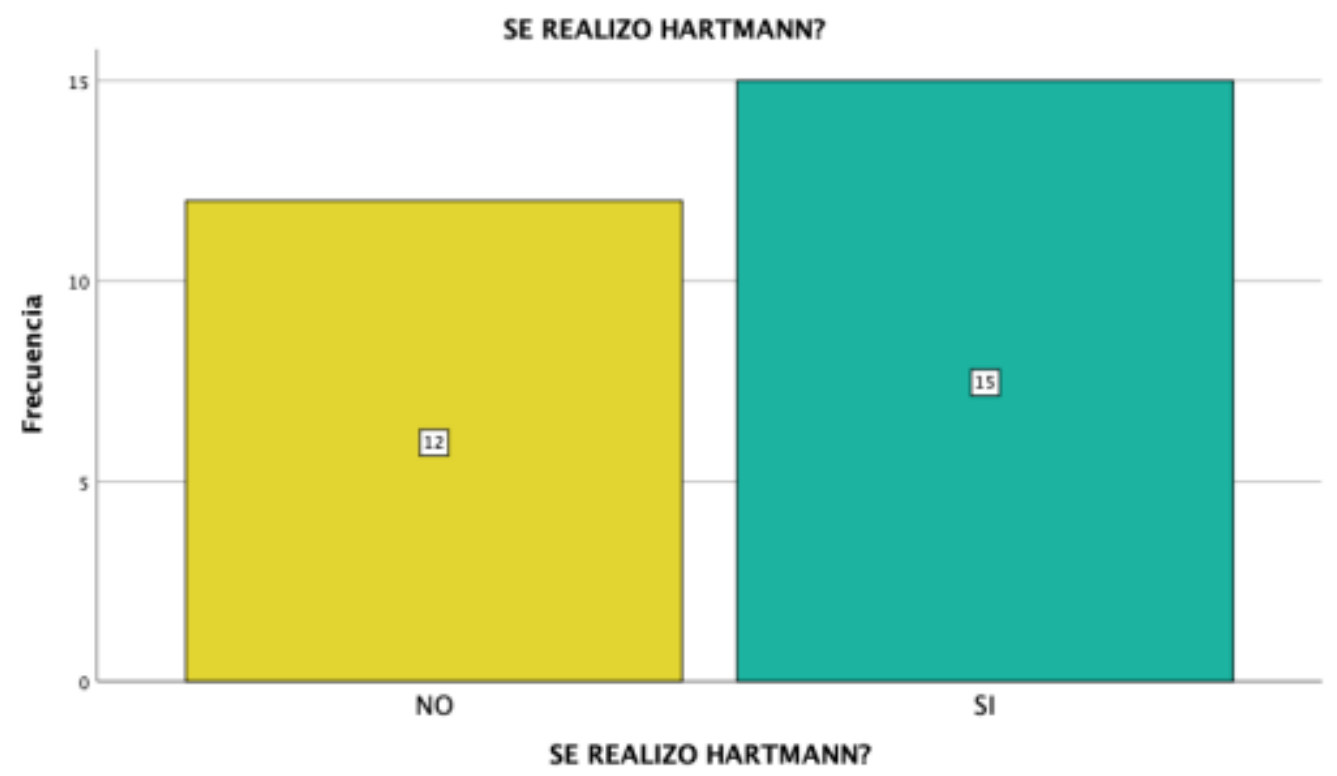

En los cinco pacientes tratados mediante lavado y drenaje laparoscópico la media de edad fue de 54.8, y en los que se realizó anastomosis primaria fue de 47 para los tres operados de urgencia y de 59 para los tres operados en forma programada.

Tabla 3.

Pacientes con complicaciones

Once pacientes presentaron complicaciones peri operatorias (morbilidad total 40,7\%). Siete de ellos correspondieron a procedimientos de Hartmann (siete de $15,46.6 \%$ ), tres con anastomosis primaria (tres de seis, $50 \%$ ) y uno con lavado y drenaje (uno de cinco, $20 \%$ ). (Tabla 3)

De las complicaciones de los procedimientos de Hartmann, se produjo una sección de ilíaca externa intraoperatoria, y en los seis restantes, cuatro de ellos presentaron sepsis mantenida con muerte (C-D 5), un seroma (C-D 1) y una infección del sitio quirúrgico (C-D 1). (Gráfico 3)

Hartmann

Otros procedimientos

Complicaciones en los procedimientos

\section{Hartmann}

Intraoperatoria

Lesión de la arteria iliaca extema

Tempranas

Sepsis

Supuración periostomal

Seroma

Tardias

Hemorragia

Otros procedimientos

Fistula enteral del drenaje

Hemoperitoneo

Peritonitis residual

Seroma $\mathrm{n}=1(9.09 \%)$

$n=6(64.55 \%)$

$\mathrm{n}=7(63,64 \%)$

$n=4(36.36 \%)$

$\mathrm{n}=1(9.09 \%)$

$\mathrm{n}=1(9.09 \%)$

$\mathrm{n}=1(9.09 \%)$ $\mathrm{n}=1(9,09 \%)$ 


\section{Gráfico 3}

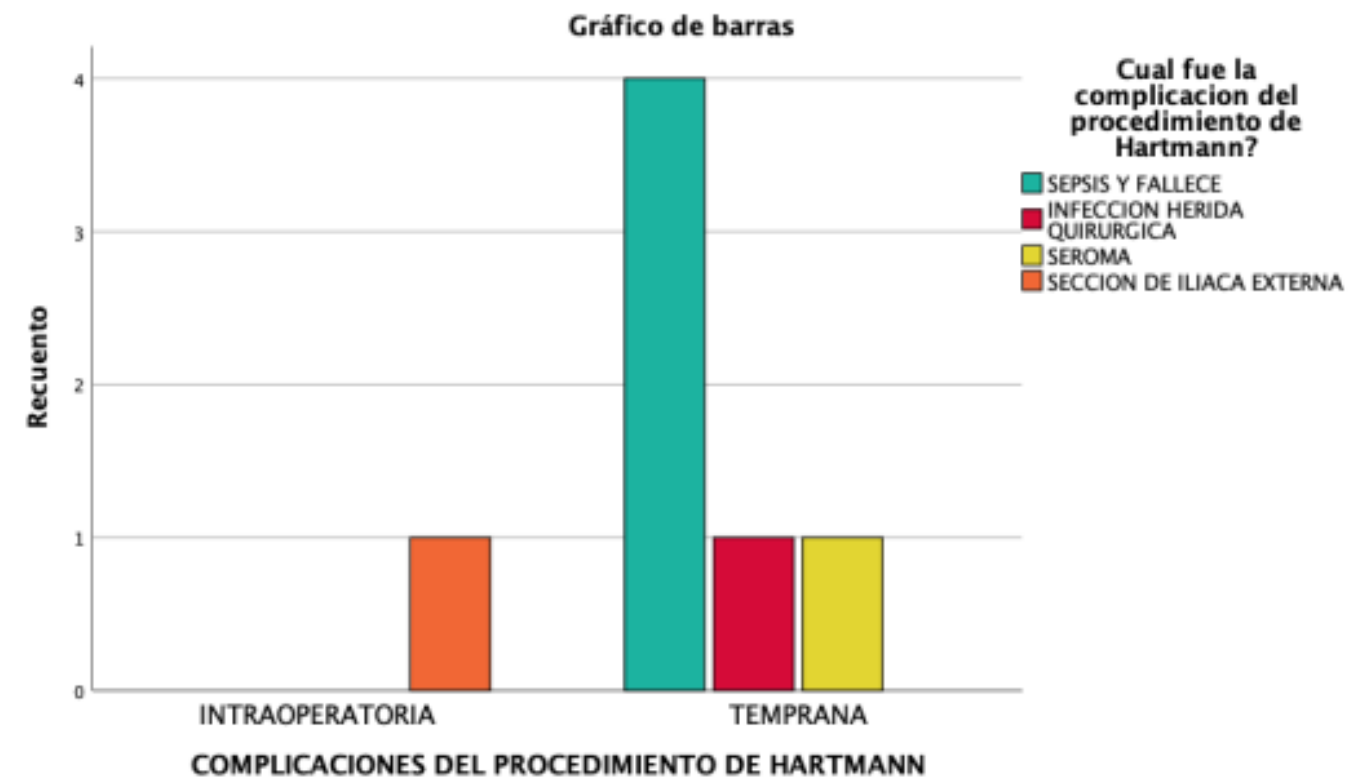

En los complicados con anastomosis primaria, las mismas fueron un hemoperitoneo (C-D 2), una peritonitis residual (C-D 2) y un seroma de la herida (C-D 1), con buena evolución en todos los casos. El paciente con lavado y drenaje laparoscópico presentó una fístula enteral (C-D 2) que se manejó en forma conservadora con evolución favorable.

No están consignadas complicaciones de la colostomía en los casos de procedimientos de Hartmann, de los cuales sobrevivieron tres de siete pacientes, ni tampoco en el caso de una colostomía de protección ante una anastomosis colo-cólica primaria

La mortalidad total fue de cuatro pacientes $(14.8 \%)$, en todos los casos se trató de procedimientos de Hartmann.

La media de estadía hospitalaria para los pacientes intervenidos por operación de Hartmann fue de 18,64 \pm 8,3 días. La media de estadía hospitalaria de anastomosis de urgencia fue de 9,5. De las de coordinación fue 10 días. El tiempo de estadía para los pacientes con drenaje laparoscópico sin resección fue de 10,5. En el único caso del paciente en el cual se realizó una resección con caño de escopeta la estadía fue de 18 días. (Tabla 4)

\section{Tabla 4.}

\section{Estadísticos}

\begin{tabular}{|c|c|c|c|}
\hline & & $\begin{array}{c}\text { Cuantos dias permanece } \\
\text { internado luego del } \\
\text { Hartmann? }\end{array}$ & $\begin{array}{l}\text { Cuantos dias permanecio } \\
\text { internado luego de realizarse } \\
\text { un procedimiento que no es } \\
\text { hartmann? }\end{array}$ \\
\hline \multirow[t]{2}{*}{$\mathrm{N}$} & Válido & 11 & 9 \\
\hline & Perdidos & 16 & 18 \\
\hline \multicolumn{2}{|c|}{ Media } & 18,64 & 11,22 \\
\hline \multicolumn{2}{|c|}{ Desv. Desviación } & 27,536 & 3,346 \\
\hline \multicolumn{2}{|c|}{ Mínimo } & 1 & 8 \\
\hline \multicolumn{2}{|c|}{ Máximo } & 92 & 18 \\
\hline
\end{tabular}


De los once pacientes sometidos al procedimiento de Hartmann que sobrevivieron, seis de ellos $(54,54 \%)$ fueron reconstruidos, en una media de tiempo de 18,3 \pm 10 meses. (Tabla 5)

Tablas cruzadas

\begin{tabular}{|c|c|c|c|c|c|}
\hline \multicolumn{6}{|c|}{$\begin{array}{c}\text { Tabla cruzada SE REALIZO HARTMANN?*SE REALIZO } \\
\text { RECONSTRUCCION DEL TRANSITO }\end{array}$} \\
\hline & & & \multicolumn{2}{|c|}{$\begin{array}{l}\text { SE REALIZO } \\
\text { RECONSTRUCCION DEL } \\
\text { TRANSITO }\end{array}$} & \multirow[b]{2}{*}{ Total } \\
\hline & & & No & SI & \\
\hline \multirow{2}{*}{$\begin{array}{l}\text { SE REALIZO } \\
\text { HARTMANN? }\end{array}$} & NO & Recuento & 11 & 1 & 12 \\
\hline & SI & Recuento & 9 & 6 & 15 \\
\hline Total & & Recuento & 20 & 7 & 27 \\
\hline
\end{tabular}

\section{Tabla 5.}

No hubo mortalidad en los pacientes reconstruidos. Tres presentaron complicaciones (morbilidad $=$ 50\%). Uno de los casos presentó una falla de sutura (C-D 4). Este paciente debió ser re-intervenido, volviendo a realizarse un procedimiento de Hartmann con buena evolución. Posteriormente se volvió a reconstruir, siendo protegida la anastomosis con una colostomía transversa, que posteriormente fue cerrada exitosamente. Este paciente presentó también una eventración del sitio de la colostomía que luego fue reparada. Otro paciente presentó una infección del sitio quirúrgico (C-D 1), con evolución favorable con tratamiento conservador. Finalmente, el tercer paciente, presentó como complicación una eventración que fue resuelta con una plastia con malla en forma tardía, también con evolución favorable.

El paciente en el que se realizó una anastomosis primaria con una colostomía de detransitacion, al ser reconstruido presentó una falla de sutura (C-D 4), con peritonitis fecaloidea, debiendo ser laparostomizado y requiriendo nuevamente una colostomía. Este paciente fue dado de alta, pero fue perdido en su seguimiento posterior.

El paciente con fístula colovesical, con resección y anastomosis colorrectal primaria y colostomía de detransitación, fue reconstruido a los 6 meses con buena evolución.

\section{Discusión}

En el presente trabajo se estableció como objetivo conocer la realidad del tratamiento quirúrgico de la enfermedad diverticular complicada en nuestro hospital, en un período reciente de 10 años, entre 2009 y 2018. Concretamente, y a la luz de publicaciones recientes y guías ${ }^{15}$, que recomiendan tratamientos hasta hace relativamente poco tiempo desaconsejados, como la anastomosis primaria de urgencia y el lavado peritoneal sin resección, nos interesaba conocer la vigencia del viejo procedimiento de Hartmann.

En primer lugar, debemos definir conceptos cuando nos referimos a enfermedad diverticular o diverticulosis complicada. Tradicionalmente se define la Diverticulosis como la presencia de los 
divertículos, la Diverticulitis como la forma sintomática secundaria a la inflamación aguda de los divertículos y la Enfermedad Diverticular complicada o Diverticulosis complicada a la presencia de complicaciones infecciosas (abscesos y/o peritonitis), fístula, obstrucción o hemorragia. De acuerdo a lo establecido en las guías de la ASCRS $2020{ }^{15}$ deben diferenciarse los términos diverticulitis complicada, diverticulitis no complicada y enfermedad diverticular sintomática no complicada. A los efectos de dichas guías se define la diverticulitis complicada como aquella que se asocia a perforación colónica libre, no contenida, con respuesta inflamatoria sistémica (SIRS), fístula, absceso, estrictura u obstrucción. La micro perforación con escasa cantidad de gas extracolónico contenido, sin SIRS, no se considera diverticulitis complicada. Diverticulitis no complicada se define como aquella con signos inflamatorios clínicos e imagenológicos, no asociada a ninguno de los elementos mencionados para la diverticulitis complicada. Finalmente se define la enfermedad diverticular sintomática no complicada, a la presencia de diverticulosis con dolor abdominal crónico, pero sin signos inflamatorios de colitis.

En cuanto al presente estudio, se obtuvieron datos a partir de la revisión de 31 historias clínicas. De estas, cuatro fueron excluidas, dado que no cumplían con los criterios de inclusión acordados. Se obtuvo un " $n$ " total de 27 historias clínicas de pacientes intervenidos quirúrgicamente por diverticulosis colónica durante un período de diez años, siendo el promedio anual de dos a tres pacientes operados por esta causa en el Hospital de Clínicas de Montevideo.

En nuestra serie la afectación de ambos sexos fue similar, predominando levemente en el sexo femenino.

Si bien se encontró una media de edad de 59,2, debemos destacar que dentro de la muestra se incluyeron dos pacientes de corta edad ( 21 y 37 años) hecho poco frecuente en esta patología. El $66,7 \%$ de los pacientes sobrepasaba los 60 años.

Veintisiete pacientes fueron operados por una enfermedad diverticular complicada con diferentes indicaciones. Dentro de ellas, la causa más frecuente (22 de 27) fue la complicación infecciosa con diferentes grados de severidad, predominando ampliamente la categoría Hinchey III, siendo casi la mitad los pacientes, seguido por Hinchey IV.

Por otro lado, solamente cinco pacientes, fueron intervenidos por sintomatología asociada a una complicación no infecciosa de esta enfermedad, como lo son las fístulas, estenosis y enterorragia. Esto es coincidente con lo publicado en la bibliografía al respecto ${ }^{16-18}$.

En aquellos pacientes intervenidos de urgencia, se realizaron distintos procedimientos: resección con procedimiento de Hartmann, resección con anastomosis primaria, en algún caso con colostomía de detransitación, resección con colostomía a dos cabos, no resección, con toilette y drenaje laparoscópico. La resección sigmoidea fue el procedimiento más utilizado ( 22 de 27 pacientes, 81 $\%)$. Los cinco pacientes en los que no se realizó una resección correspondieron a paciente con un Hinchey I, los tres con Hinchey II y un paciente con un Hinchey III, pero en el que no se logró objetivar el sitio de la perforación sigmoidea. En todos estos casos el tratamiento realizado fue la exploración laparoscópica, con toilette y drenaje. A pesar del gran desarrollo del abordaje video asistido y de la promoción del lavado y drenaje sin resección en la literatura ${ }^{19-21}$, la resección, seguida del procedimiento de Hartmann sigue siendo la opción predominante en estos casos, alcanzando el $62.5 \%$ del total de nuestra serie. La explicación de este predominio se fundamenta en que en su 
mayoría se trató de casos evolucionados, en pacientes graves, tanto por sus comorbilidades como por el compromiso infeccioso sistémico, en donde otras opciones fueron consideradas menos seguras. Es de destacar también que el análisis fue realizado exclusivamente de pacientes operados, excluyendo a todos los pacientes que fueron tratados médicamente, con o sin internación, y con o sin drenajes percutáneos guiados por imagen, lo cual supone la opción terapéutica dominante en el momento actual para los casos moderados ${ }^{22}$. Esto se refleja también en el elevado porcentaje de casos Hinchey III y IV, es decir, con peritonitis difusas de severidad variable, en las que la opción de una toilette laparoscópica resulta muy engorrosa y prolonga la cirugía, lo que en definitiva terminó descartando dicha posibilidad, con la excepción del caso comentado.

La mortalidad global fue de $14.8 \%$ correspondiendo en todos los casos a pacientes en los que se realizó una operación de Hartmann. Dado el tamaño muestral no fue posible realizar el ajuste estadístico, pero se trató siempre de los casos más graves, por la edad, las comorbilidades y la severidad y evolución del cuadro agudo. Lo mismo explicaría la morbilidad, siendo la global de un $40.7 \%$, pero que en el caso del Hartmann alcanza casi la mitad de los pacientes. Los casos de abordaje laparoscópico con lavado y drenaje, no presentaron mortalidad y la morbilidad fue inferior, de un 20 $\%$, pero se trató de pacientes en general seleccionados, con menor riesgo previo y cuadros menos evolucionados. De todos modos, la mortalidad global de la serie es aceptable en comparación con series similares publicadas, al igual que sucede con la morbilidad ${ }^{23-27}$. A su vez, la mayoría de las complicaciones fueron leves, vinculadas al sitio quirúrgico. Los cuatro pacientes con peritonitis fecaloideas y sepsis refractaria fueron los fallecidos. El resto de los pacientes presentaron una evolución favorable, incluyendo una infrecuente lesión incidental de la arteria ilíaca externa. La inmensa mayoría fueron complicaciones inmediatas que prolongaron la estadía hospitalaria, destacando un único caso de complicación tardía como fue una supuración paracolostómica. Debemos destacar que prácticamente no se consignaron complicaciones vinculadas al estoma, hecho llamativo y que hubiera sido esperable, dado las dificultades vinculadas a su confección en la urgencia, especialmente en pacientes obesos.

Si analizamos la estadía hospitalaria, la media fue de 18 días para la técnica de Hartmann, claramente superior al resto de los procedimientos, siendo la explicación la misma que para la morbimortalidad, por tratarse justamente de los pacientes más graves. Incluso en el caso del abordaje laparoscópico sin resección, la estadía hospitalaria fue considerable, de una media de 10.5 días, también en línea con la gravedad de la patología y el tipo de pacientes.

La reconstrucción del tránsito digestivo se realizó en seis de los pacientes (54,5\%) que sobrevivieron luego de la operación de Hartmann $(n=11)$, en concordancia con las cifras consultadas en la bibliografía ${ }^{28-30}$. A pesar que no existe un consenso sobre el tiempo esperado para realizar la reconstrucción del tránsito intestinal, es recomendada su realización en tiempo alejado (3 a 9 meses) $)^{31,32}$. La media de tiempo en nuestra muestra fue de 18 meses, no conocemos los motivos por los cuales este periodo fue tan extenso. Estos pacientes, fueron en su totalidad menores a 73 años. Se sabe que una edad mayor 80 es considerado un factor de riesgo importante de morbimortalidad en la evaluación de la reconstrucción intestinal. Aquellos no reconstruidos, tuvieron como motivo el mal terreno y riesgo anestésico-quirúrgico, fallecimiento por otra causa, o la pérdida de seguimiento.

La tasa de complicaciones en la reconstrucción luego de Hartmann fue de 50\%, lo que coincide con la bibliografía consultada ${ }^{33-35}$. El alto nivel de complicaciones en la reconstrucción del tránsito luego 
de Hartmann lleva a una alta mortalidad, aunque en este caso, no se presentaron fallecimientos. De las complicaciones la más grave fue la falla de sutura, presentándose en un solo paciente, quien fue re-intervenido mediante Hartmann procediendo meses después a la restauración del tránsito intestinal. Dada la edad (57 años) y el buen terreno anestésico quirúrgico del paciente fueron posibles este re intervenciones con un resultado exitoso.

En suma, en nuestra serie el procedimiento de Hartmann fue la técnica más utilizada en pacientes intervenidos de urgencia por enfermedad diverticular colónica complicada. La morbimortalidad fue similar a lo publicado en la mayoría de las series internacionales ${ }^{36}$. La tasa de reconstrucción fue de aproximadamente la mitad, lo que es coincidente con lo analizado en la bibliografía ${ }^{37,38}$. Ello se debe a que se trata de pacientes añosos, con muchas comorbilidades que una vez superado el proceso agudo no se justifica la re-intervención alejada.

\section{Conclusiones}

Podemos concluir que la operación de Hartmann mantiene vigencia en el tratamiento quirúrgico de esta enfermedad, la cual, si bien es muy prevalente, el avance y desarrollo del tratamiento médico y de los procedimientos mini-invasivos, han llevado a que el número de cirugías descienda. Los pacientes en definitiva operados son en general cuadros graves, por su edad y comorbilidad y por lo evolucionado del cuadro, lo cual hace que la opción por un procedimiento de Hartmann siga siendo la más frecuente, lo cual es concordante con todo lo publicado recientemente. Las opciones tanto de anastomosis primaria como de lavado y drenaje sin resección, si bien evitan la morbimortalidad y afectación de la calidad de vida asociada al Hartmann, siguen siendo controversiales, no sustentadas en evidencia de primer nivel, y deben ser reservadas para casos seleccionados.

\section{Referencias}

1 Wilkins T, Embry K, George R. Diagnosis and Management of Acute Diverticulitis. Am Fam Phys 2013; 87 (9): 612-20

2. García-osogobio JMCS. Sigmoidectomía con anastomosis primaria para diverticulitis complicada. Rev Gastroenterol México [Internet]. 2015;80(4):255-9.

http://dx.doi.org/10.1016/j.rgmx.2015.07.005

3. Chabok A, Pahlman L, Hiern F, Haapaniemi S Smedh K, AVOD study group. Randomized clinical trial of antibiotics in acute uncomplicated diverticulitis. Br J Surg. 2012; 99:532-9.

4. Daniels L, Unlu C, de Korte N, van Dieren S, Stockmann HB, Vrouenraets BC, Consten EC, et al. Randomized clinical trial of observational versus antibiotic treatment for a first episode of CTproven uncomplicated acute diverticulitis. Br J Surg. 2017; 104:52-61. 
5. Ambrosetti P. Acute diverticulitis of the left colon: value of the initial CT and timing of elective colectomy. J Gastrointest Surg.2008;12:1318-1320. doi: 10.1007/s11605-008-0489-8

6. Ambrosetti P, Becker C, Terrier F. Colonic diverticulitis: impact of imaging on surgical management - a prospective study of 542 patients. Eur Radiol. 2002; 12:1145-1149 doi: $10.1007 /$ s00330-001-1143-y

7. Rosado R, Blasco T, Ferrer M, Marín H, Pérez-Domínguez L, Biondo S, Roig-Vila JV. Enfermedad diverticular complicada: toma de posición sobre tratamiento ambulatorio, intervención de Hartmann, lavado-drenaje peritoneal y cirugía laparoscópica. Documento de consenso de la Asociación Española de Coloproctología y Sección de Coloproctología de la Asociación Española de Cirujanos. Cir Esp 2017; 95(7): 369-77. doi: 10.1016/j.ciresp.2017.03.008

8. Cirocchi R, Afshar S, Di Saverio S, Popivanov G, De Sol A, Gubbiotti F, Tugnoli G, et al. A historical review of surgery for peritonitis secondary to acute colonic diverticulitis: from LockhartMummery to evidence-based medicine. World Jour Em Surg 2017; 14: 1-25. Doi: 10.1186/s13017017-0120-y

9. Halim H, Askari A, Nunn R, Hollingshead H. Primary resection anastomosis versus Hartmann's procedure in Hinchey III and IV diverticulitis. World Jour Em Surg 2019; 32: 4-11. doi: 10.1186/s13017-019-0251-4.

10. Charúa-Guindic L, Jiménez-Bobadilla B, Reveles-González A, Avendaño-Espinosa O, CharúaLevy E. Incidencia, diagnóstico y tratamiento de la fístula colovesical. Cir Cir 2007;7575(5):343-9. Disponible en: http://www.medigraphic.com/pdfs/circir/cc-2007/cc075e.pdf

11. Resio BJ, Jean R, Chiu AS, Pei KY. Association of Timing of Colostomy Reversal With Outcomes. JAMA Surg. 2019; 154 (3): 218-224. doi: 10.1001 / jamasurg.2018.4359

12. Javier F, Fernández M, Jiménez ND, Belén A, Herrera G, Luque IG, Garcilazo Arsimendi DJ, Gómez Barbadillo J. Nuevas tendencias en el manejo de la diverticulitis y la enfermedad diverticular del colon. Rev Esp Enf Dig 2015; 107:162-70

13. Dindo D, Demartines N, Clavien P-A. Classification of Surgical Complications. A New Proposal With Evaluation in a Cohort of 6336 Patients and Results of a Survey. Ann Surg 2004; 240(2): 205-13. doi: 10.1097/01.sla.0000133083.54934.ae

14. Clavien P-A, Barkun J, L de Oliveira M, Vauthey JN, Dindo D, Schulick R, de Santibañes E, et al. The Clavien-Dindo Classification of Surgical Complications. Five-Year Experience. Ann Surg 2009; 250(2): 187-96. doi: 10.1097/SLA.0b013e3181b13ca2

15. Hall J, Hardiman K, Lee S, Lightner A, Stocchi L, Paquette I, Steele S, et al. The American Society of Colon and Rectal Surgeons Clinical Practice Guidelines for the Treatment of Left-Sided Colonic Diverticulitis. Dis Colon Rectum 2020; 63: 728-747.doi:10.1097/DCR.0000000000001679

16. Wieghard N, Geltzeiler CB, Tsikitis VL. Trends in the surgical management of diverticulitis. Ann Gastroenterol. 2015;28(1):25-30 
17. Lambrichts DP, Birindelli A, Tonini V, Cirocchi R, Cervellera M, Lange JF, Bemelman W, et al. The Multidisciplinary Management of Acute Complicated Diverticulitis. Inflam Intest Diseases 2018; 3: 80-90. doi: 10.1159/000486677

18. Deery SE, Hodin RA. Management of diverticulitis in 2017. J Gastrointest Surg. 2017; 21:1732-1741. doi: 10.1007/s11605-017-3404-3

19. Thornell A, Angenete E, Gonzales E, Heath J, Jess P, Läckberg Z, Ovesen H, et al. Treatment of acute diverticulitis laparoscopic lavage vs. resection (DILALA): study protocol for a randomised controlled trial Treatment of acute diverticulitis laparoscopic a randomised controlled trial. Trials 2011;186:1-6. doi: 10.1186/1745-6215-12-186

20. Ceresoli M, Coccolini F, Montori G, Catena F, Sartelli M, Ansaloni L. Laparoscopic lavage versus resection in perforated diverticulitis with purulent peritonitis: a metaanalysis of randomized controlled trials. World Journ Em Surg 2016; 42: 1-8. doi: 10.1186/s13017-016-0103-4

21. Vennix S, Musters G, Mulder I, Swank H, Consten E, Belgers E, van Geloven A, et al. Laparoscopic peritoneal lavage or sigmoidectomy for perforated diverticulitis with purulent peritonitis: a multicentre, parallel-group, randomised, open-label trial. Lancet. 2015; 386:1269-77. Doi: 10.1016/S0140-6736(15)61168-0

22. Lambrichts DPV, Bolkenstein HE, van der Does DCHE, Dieleman D, Crolla R M P H, Dekker J W T, van Duijvendijk P, et al. Multicentre study of non-surgical management of diverticulitis with abscess formation. Br J Surg. 2019; 106:458-466 doi: 10.1002/bjs.11129

23. Santander C, Astudillo P, Manterola C. Procedimiento de Hartmann vs resección y anastomosis primaria en peritonitis diverticular de colon izquierdo por cirugía abierta. Revisión sistemática de la literatura. Rev Chil Cir 2013;65:271-8 doi: 10.4067/S0718-40262013000300014

24. Garber A, Hyman N, Osler T. Complications of Hartmann takedown in a decade of preferred primary anastomosis. Am J Surg [Internet] 2014;207(1):60-4. Doi: 10.1016/j.amjsurg.2013.05.006

25. Hupfeld L, Burcharth J, Pommergaard H, Rosenberg J. The Best Choice of Treatment for Acute Colonic Diverticulitis with Purulent Peritonitis Is Uncertain. BioMed Research International 2014: 1-5 Article ID 380607. Doi: 10.1155/2014/380607

26. Biondo S, Borao JL, Millan M, Kreisler E et al. Current status of the treatment of acute colonic diverticulitis: a systematic review. Colorectal Dis 2011; 14(1): 1-11. Doi: 10.1111/j.14631318.2011.02766.x

27. Bridoux V, Regimbeau JM, Ouaissi M, Mathonnet M, Mauvais F, Houivet E, Schwarz L, Mege D, et al. Hartmann's procedure or primary anastomosis for generalized peritonitis due to perforated diverticulitis: a prospective multicenter randomized trial (DIVERTI). J Am Coll Surg. 2017; 225:798-805. doi: 10.1016/j.jamcollsurg.2017.09.004

28. Gachabayov M, Oberkofler CE, Tuech JJ, Hahnloser D, Bergamaschi R. Resection with primary anastomosis vs nonrestorative resection for perforated diverticulitis with peritonitis: a systematic review and meta-analysis. Colorectal Dis. 2018; 20:753-770. doi: 10.1111/codi.14237 
29. Acuna SA, Dossa F, Baxter NN. The end of the Hartmann's era for perforated diverticulitis. Lancet Gastroenterol Hepatol. 2019; 4:573-575. doi: 10.1016/S2468-1253(19)30182-7

30. Lee JM, Bai P Chang J, El Hechi M, Kongkaewpaisan N, Bonde A, Mendoza A, Saillant N, et al. Hartmann's procedure vs primary anastomosis with diverting loop ileostomy for acute diverticulitis: nationwide analysis of 2,729 emergency surgery patients. J Am Coll Surg. 2019; 229:48-55. doi: 10.1016/j.jamcollsurg.2019.03.007

31. Moro-Valdezate D, Royo-Aznar A, Martín-Arévalo J, Pla-martí V, García-Botello S, Espinoza SL Fernández-Moreno MC et al. Outcomes of Hartmann's procedure and subsequent intestinal restoration. Which patients are most likely to undergo reversal? Am Journ Surg 2019(11), 218(5): 918-27. DOI: 10.1016/j.amjsurg.2019.02.025

32. Roig V, Salvador A, Frasson M, García L, Espinosa J, Roselló V, Hernandis J et al. Stoma Reversal After Surgery for Complicated Acute Diverticulitis: A Multicentre Retrospective. Cir Esp 2018; 6:2-10 doi: /10.1016/j.cireng.2018.02.012

33. Sherman KL, Wexner SD, Ed F. Considerations in Stoma Reversal. Clinics in Colon and Rectal Surgery 2017; 172-7. doi: 10.1055/s-0037-1598157

34. Sciuto P, Rappa J, Meineri J, Ruso L, Rodríguez Temesio G. Restitución del procedimiento de Hartmann por vía laparoscópica. Análisis de nuestra experiencia en el Hospital Maciel de Montevideo, Uruguay, Clínicas Quirúrgicas 2 y 3 de la Facultad de Medicina de la Universidad de la República (UDELAR). Rev Chil Cir 2017;69(6): 446-51. Doi: 10.1016/j.rchic.2017.06.001

35. Rosenthal R, Kettelhack C, Oertli D. Reversal after Hartmann's procedure in patients with complicated sigmoid diverticulitis. Colorectal Dis 2016; 19(6): 582-8 doi: 10.1111/codi.13553

36. Aquina CT, Probst CP, Becerra AZ. Colon / rectum The impact of surgeon volume on colostomy reversal outcomes after Hartmann' s procedure for diverticulitis. Surgery [Internet].2016;160(5):1309-17. Doi: 10.1016/j.surg.2016.05.008

37. Hallam S, Mothe BS, Tirumulaju RMR. Hartmann's procedure, reversal and rate of stoma-free survival. Ann R Coll Surg Engl 2018; 100: 301-7 doi 10.1308/rcsann.2018.0006

38. Zarnescu EC, Zarnescu NO, Costea R, Rahau L, Neagu S. Morbidity after reversal of Hartmann operation: retrospective analysis of 56 patients. J Med Life 2015;8(4):488-91 
Anexo 1. Clasificación de Claiven y Dindo.

\begin{tabular}{|c|c|c|}
\hline Grado & & Definición \\
\hline I & & $\begin{array}{l}\text { Cualquier desviación del postoperatorio normal que no requiera } \\
\text { reintervención a cielo abierto ni endoscópica. Se considera el incluir } \\
\text { el uso de soluciones electroliticas, antiométicos, antipiréticos, } \\
\text { analgésicos y fisioterapias. Incluye infección superficial tratada en la } \\
\text { cama del paciente. }\end{array}$ \\
\hline II & & $\begin{array}{l}\text { Se requiere tratamiento farmacologico diferente a los anteriores. Uso } \\
\text { de transfusiones sanguineas o de hemoderivados y nutrición } \\
\text { parenteral. }\end{array}$ \\
\hline \multirow[t]{3}{*}{ III } & & Requiere reintervención quirúrgica endoscópica o radiologica \\
\hline & a & Sin anestesia general. \\
\hline & b & Con anestesia general. \\
\hline \multirow[t]{3}{*}{ IV } & & $\begin{array}{l}\text { Complicaciones que amenazan la vida del paciente y requieren } \\
\text { tratamiento en cuidados intermedios o intensivos. }\end{array}$ \\
\hline & $\mathbf{a}$ & Disfunción orgánica única (Incluye la diálisis). \\
\hline & b & Disfunción orgánica múltiple. \\
\hline $\mathrm{V}$ & & Muerte del paciente. \\
\hline
\end{tabular}

Sufijo d, es cuando el paciente sufre una complicación al alta, se coloca este sufijo ante el grado.

(Tomado de la traducción realizada por Monterola ") 